Check for updates

Cite this: RSC Adv., 2017, 7, 31264

Received 15th May 2017

Accepted 12th June 2017

DOI: $10.1039 / c 7 r a 05469 f$

rsc.li/rsc-advances

\section{High energy conversion efficiency conducting polymer actuators based on PEDOT:PSS/MWCNTs composite electrode $\uparrow$}

\author{
Dongxing Wang, ${ }^{\text {ab }}$ Chao Lu, ${ }^{\mathrm{b}}$ Jingjing Zhao, ${ }^{\mathrm{b}}$ Song Han, ${ }^{\mathrm{b}}$ Minghong $\mathrm{Wu}^{\mathrm{a}}$ \\ and Wei Chen (iD)*b
}

Conducting polymer actuators have attracted enormous attention due to their capacity to be driven by lowvoltage, their large deformation, and ability to work in air. However, they usually present low conversion efficiency, cycle stability and response actuation speed which severely hamper their practical application. Here, we report a high performance conducting polymer actuator based on a PEDOT:PSS/MWCNTS electrode, which displays high actuation performance, including high electromechanical conversion efficiency $(1.04 \%)$, large actuation strain $(0.64 \%)$, high strain rates $\left(8.83 \% \mathrm{~s}^{-1}\right)$, excellent cycle stability (100 000 cycles), and good generated blocking force $(1.43 \mathrm{mN})$ under $2.5 \mathrm{~V}$. The key to the higher performance lies in the porous structure, high electrical conductivity $\left(153.8 \mathrm{~S} \mathrm{~cm}^{-1}\right.$ ) and mechanical strength $(918.3 \mathrm{MPa})$ of the PEDOT:PSS electrode coupled with carboxylic MWCNTs, which result in a great improvement of ion storage capacity in the actuator. Our studies demonstrate the important role of MWCNTs in enhancing the performances of conducting polymer actuators, which will guide the development of next generation electroactive polymer actuators.

\section{Introduction}

In past decades, ionic electroactive polymer (EAP) actuators based on ionic polymer metal composites (IPMC), conducting polymers and carbon nanomaterials have been widely studied due to their impressive actuation performance. ${ }^{1-3}$ Among them, conducting polymers, such as polypyrrole, polythiophene and polyaniline, have been regarded as promising candidates for the preparation of lightweight, flexible and biocompatible EAP actuators. Actuators based on CPs, which dimensional changes resulting from electrochemical doping, transportation of dopant ions between the polymer matrix and the surrounding electrolyte solution, have the following advantages: combination of low driving voltage (typically 1-3 V), large deformation and fast response speed compared with traditional IPMC. ${ }^{4-6}$

Poly(3,4-ethylenedioxythiophene):poly(styrenesulfonic acid) (PEDOT:PSS) is considered as one of most successful commercially available conducting polymers owing to its high transparency, high flexibility, and excellent thermal stability. However, its low conductivity and mechanical strength have been limiting factors for practical applications. The electrical

${ }^{a}$ Department of Chemistry, College of Sciences, Shanghai University, Shanghai 200444, P. R. China

${ }^{b} i$-Lab, Suzhou Institute of Nano-Tech and Nano-Bionics, Chinese Academy of Sciences, Suzhou 215123, P. R. China. E-mail: wchen2006@sinano.ac.cn

$\dagger$ Electronic supplementary information (ESI) available. See DOI: $10.1039 / \mathrm{c} 7 \mathrm{ra} 05469 \mathrm{f}$ conductivity of PEDOT:PSS can be improved by various of approaches. Recent studies have found that the conductivity of PEDOT:PSS can be significantly enhanced treating by thermal, organic solvents, ionic liquids, surfactants and acids..$^{711}$ Kim et al. reported the impressive high conductivity of PEDOT:PSS up to $4380 \mathrm{~S} \mathrm{~cm}^{-1}$ by $\mathrm{H}_{2} \mathrm{SO}_{4}$ treatment, which achieved over four orders of magnitude enhancement compared with untreated PEDOT:PSS. ${ }^{12}$ Besides the above mentioned treatment method, it is highly desirable to add an additional additive into PEDOT:PSS. Such an additive is required to own excellent conductivity and mechanical rigidity to improve the properties of pristine PEDOT:PSS. Recently, carbon nanotubes, which first discovered by Iijima, ${ }^{13}$ are regarded as a good additive for PEDOT:PSS due to their superb electrical, mechanical and chemical properties. ${ }^{14-16}$ Terasawa et al. developed PEDOT:PSS/ SWCNT/IL actuator which combining electrostatic double-layer and faradaic capacitors that exhibited more superior electrochemical characteristics and actuation performance compared with PEDOT:PSS/IL actuator. ${ }^{17}$

In this work, PEDOT:PSS electrode doping with various content of carboxylic MWCNTs were prepared by solutioncasting method and characterized by means of a four-point technique, FE-SEM and tensile test etc. The doping of carboxylic MWCNTs generates conjugate effect and hydrogen bonding with PEDOT:PSS, which not only obviously changes polymer structure but also enhances the electrical conductivity and mechanical properties of the electrodes. Furthermore, we fabricated three-layer structure actuator (as shown in Fig. 1) 
$\mathbf{a}$

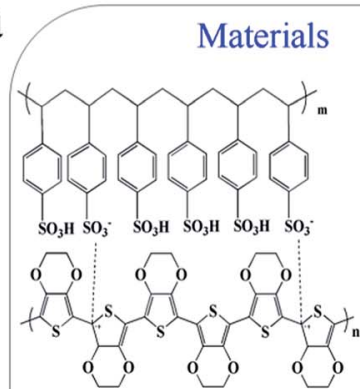

PEDOT:PSS

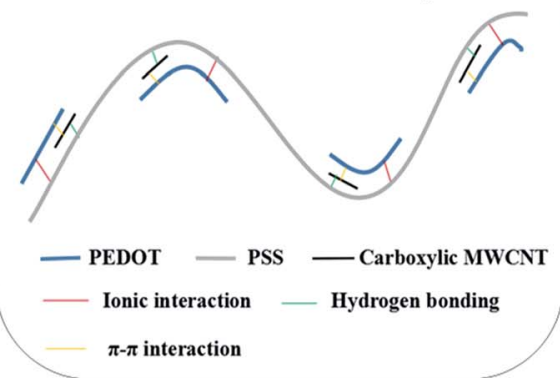

b

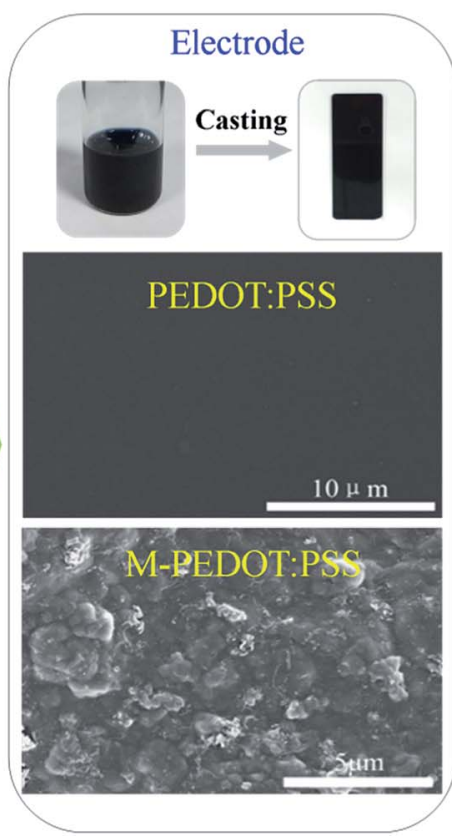

c

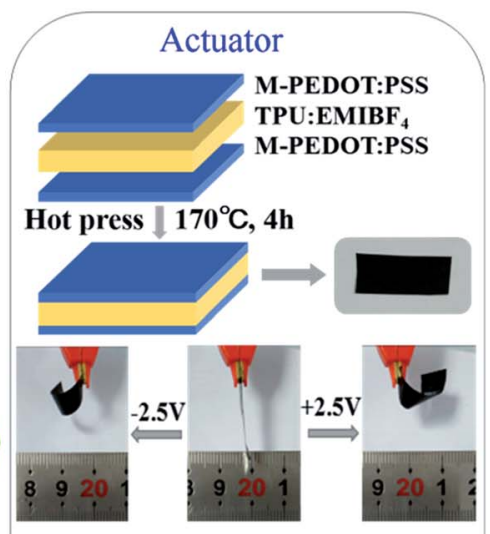

Section of actuator

Fig. 1 Schematic illustration of actuator based on M-PEDOT:PSS electrode and fabricated by hot press.

taking $\mathrm{TPU} / \mathrm{EMIBF}_{4}$ as supporting polymer. Benefit from the porous structure and high electric conductivity of composite electrode, the obtained actuators based on PEDOT:PSS/ MWCNTS (M-PEDOT:PSS) electrode displayed a greatly improved actuation performance compared with pristine PEDOT:PSS actuator. The actuator based on M-PEDOT:PSS electrode were capable of producing large bending deformation (up to the strain of $0.64 \%)$, high strain rates $\left(8.83 \% \mathrm{~s}^{-1}\right)$, high electromechanical conversion efficiency $(1.04 \%)$ and enhanced force generation $(1.43 \mathrm{mN})$ at $2.5 \mathrm{~V}$.

\section{Experimental section}

\subsection{Materials}

PEDOT:PSS (Clevios, PH1000, 1.3 wt\%) was commercially available in the form of water dispersion form Heraeus. The carboxylic MWCNTs (95\%, XF NANO, Inc.) had an - $\mathrm{COOH}$ content $3.86 \mathrm{wt} \%$, average length of $10-30 \mu \mathrm{m}$, and surface area $500 \mathrm{~m}^{2} \mathrm{~g}^{-1}$. Fig. 1a shows the chemical structures of PEDOT:PSS and carboxylic MWCNTs. Thermoplastic polyurethane (TPU) was obtained from DuPont ${ }^{\mathrm{TM}}$. 1-Ethyl-3-methyl imidazolium tetra-fluoroborate $\left(\mathrm{EMIBF}_{4}, 99 \%\right)$ purchased from Shanghai Cheng Jie Chemical Co. $N, N$-Dimethylformamide (DMF) were obtained from Sinopharm Chemical Reagent Co., Ltd and deionized water was purified through Ultrapure Milli-Q system.

\subsection{Preparation of electrodes layers}

The carboxylic MWCNTs was dispersed in the PEDOT:PSS solution by a magnetic stirring for $6 \mathrm{~h}$ to obtain a homogeneous solution. The content of carboxylic MWCNTs in PEDOT:PSS was 10-30 wt\%, and it was then homogenized for $2 \mathrm{~h}$ by bath sonication using a KQ-300DA sonicator at room temperature
(Fig. S1a, ESI †). Finally, $3 \mathrm{~mL}$ mentioned above homogeneous solution was casted on a glass substrate $(2.5 \mathrm{~cm} \times 7.5 \mathrm{~cm}$ size $)$ and dried at $60{ }^{\circ} \mathrm{C}$ for 1 day to obtained PEDOT:PSS/MWCNT (M-PEDOT:PSS) electrodes film (Fig. S1b†). The PEDOT:PSS electrodes film without carboxylic MWCNTs was fabricated in the same method. The thickness of the resulting electrodes film was about $25 \mu \mathrm{m}$.

\subsection{Preparation of the electrolyte layers}

$1 \mathrm{~g}$ TPU and $1 \mathrm{~g} \mathrm{EMIBF}$ was dissolved in $30 \mathrm{~mL} \mathrm{DMF}$ at $80^{\circ} \mathrm{C}$ overnight to obtain uniform solution. Then $2 \mathrm{~mL}$ TPU/EMIBF 4 solution was casted on a glass substrate $(2.5 \mathrm{~cm} \times 7.5 \mathrm{~cm}$ size $)$ and dried at $80{ }^{\circ} \mathrm{C}$ for 2 days to obtained TPU/EMIBF 4 electrolyte film.

\subsection{Assembly of the sandwiched actuators}

Two piece of M-PEDOT:PSS electrode films were laminated on $\mathrm{TPU} / \mathrm{EMIBF}_{4}$ electrolyte film and heat pressed at $170{ }^{\circ} \mathrm{C}$ for 4 hours and naturally cooled to room temperature. After that, the film was carefully peeled from the glass substrate to get a freestanding sandwiched membrane. The thickness of obtained actuator membranes were about $110 \mu \mathrm{m}$. Then the actuator membranes were cut into strips with a standard dimension (5 $\mathrm{mm}$ in width and $20 \mathrm{~mm}$ in length). The pristine PEDOT:PSS electrode based actuator was constructed by the same process.

\subsection{Characterization}

FE-SEM was recorded by a Hitachi S-4800. The samples were dried in the vacuum oven overnight before capturing the surface and cross-sectional images. The electrical conductivity of electrode was tested by a multifunction digital four-probe tester (ST-2258C). The mechanical properties were evaluated 
by a tensile test with a mechanical tester (AGS-X, Shimadzu), where tensile modulus, tensile strength, and elongation at break were calculated from the stress-strain curves. The blocking forces of the actuators under AC and DC step input by $2.5 \mathrm{~V}$ were measured by using a load cell (JZ-101, XINHANG).

Cyclic voltammetry (CV) was analyzed by a CHI760D in CV electrochemical work station. The specific capacitance $\left(\mathrm{F} \mathrm{g}^{-1}\right)$ measurements were calculated using the following equation: ${ }^{17}$

$$
C_{\text {sp }}=\frac{1}{\Delta V v S} \int_{V_{1}}^{V_{2}} I \mathrm{~d} V
$$

where $\Delta V$ is the potential window, $v$ is the scan rate and $S$ is the weight taken of the M-PEDOT:PSS or PEDOT:PSS electrodes of the actuators.

The tip displacement $(\delta)$ of the device was measured by a Keyence LK-G80 laser positioning system, where the strain $(\varepsilon)$ and strain rate $\left(\varepsilon_{\mathrm{r}}\right)$ of the actuators were estimated by the following equations: ${ }^{18,19}$

$$
\begin{gathered}
\varepsilon=\frac{2 d \delta}{\delta^{2}+L^{2}} \\
\varepsilon_{\mathrm{r}}=4 \varepsilon f
\end{gathered}
$$

where $d, \delta, f$ and $L$ are the thickness, tip displacement, frequency of applied potential and free length of the actuator strip, respectively. And constant 4 in equations is the number of movements between neutral and utter position during the full cycle of the applied potential.

\section{Results and discussion}

\subsection{M-PEDOT:PSS}

The chemical structure of PEDOT:PSS and carboxylic MWCNTs are shown in Fig. 1a. In the conducting polymer of PEDOT:PSS, PEDOT cations and PSS counter anions forms a poly-ion complex through electrostatic interactions. ${ }^{20}$ The schematic illustration of interaction between the PEDOT:PSS and carboxylic MWCNTs is also displayed in Fig. 1a. The coil structure PEDOT unit is attached along with the long PSS chains through ionic interaction. The carboxylic MWCNTs weak the ionic interaction between PEDOT and PSS by forming conjugate effect and hydrogen bond with PEDOT and PSS respectively. The interactions between them will bring about tiny separation between PEDOT unit and PSS chain, ${ }^{21}$ and the porous structure is formed by incorporation of MWCNTs simultaneously. The change of PEDOT:PSS micro-structure have an effect on the electrode properties such as electrical conductivity, mechanical strength and stability.

\subsection{Morphological analysis}

In order to investigate the influence of the addition of carboxylic MWCNTs on the micro-structure of the PEDOT:PSS, SEM images have been made and presented in Fig. 1b and 2. Fig. 1b revealed the surface morphology of the pristine PEDOT:PSS and M-PEDOT:PSS electrode film. It can be seen that the

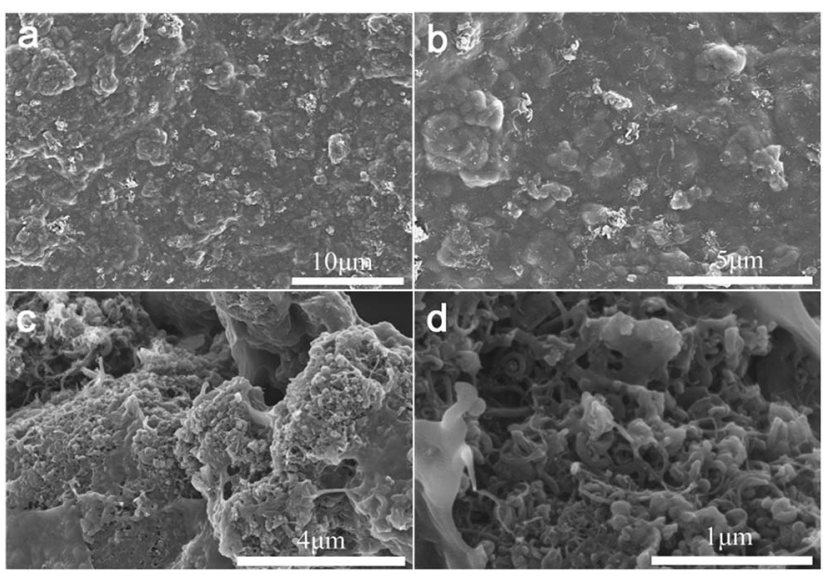

Fig. 2 ( $a$ and b) The surface morphology of the M-PEDOT:PSS electrode film at different magnification. (c and d) The internal morphology of the M-PEDOT:PSS electrode film at different magnification.

morphology of PEDOT:PSS electrode film is dense, smooth with no voids. With the addition of carboxylic MWCNTs into the PEDOT:PSS matrix, the morphology of electrode film becomes rough, porous and more voids structures are observed, as shown in Fig. 2. As presented in Fig. $2 \mathrm{a}$ and b, the surface of electrode film becomes rough. And porous and voids structures can be seen from internal of electrode film (Fig. $2 c$ and d). The carboxylic MWCNTs are homogenous distributed in the PEDOT:PSS matrix by the effect of $\pi-\pi$ interaction and hydrogen bond. By virtue of these coupling interaction, the carboxylic MWCNTs not only enhances the tensile strength and electrical conductivity but also improve the electrochemical activity of pristine PEDOT:PSS electrode.

\subsection{Electrical conductivity}

The thickness of the electrode films (Table S1, ESI $\dagger$ ) continue to increase to $28 \mu \mathrm{m}$ owing to the micro-structure of PEDOT:PSS becomes rough and porous after addition of carboxylic MWCNTs as presented in SEM images. Fig. 3a demonstrated the effect of carboxylic MWCNTs as a function of weight fraction on the electrical conductivity of the electrode films. The electrode electrical conductivity revealed a linear increase with the MWCNTs content, indicating that the electrical conductivity can be well controlled by changing the weight fraction of MWCNTs. As listed in the Table $\mathrm{S} 1, \uparrow$ the conductivity of pristine PEDOT:PSS film is only $4.64 \mathrm{~S} \mathrm{~cm}^{-1}$. By the addition of carboxylic MWCNTs, the conductivity of sample containing 10 wt $\%$ of MWCNTs is $40.17 \mathrm{~S} \mathrm{~cm}^{-1}$, which reveals a fast increase in the conductivity with the raise of MWCNTs mass fraction. Moreover, the conductivity of M-PEDOT:PSS composite film improves to $153.75 \mathrm{~S} \mathrm{~cm}^{-1}$ when the MWCNTs weight fraction reaches about $30 \mathrm{wt} \%$. It should be noted here the electrical conductivity of the $30 \%$ M-PEDOT:PSS film $\left(153.75 \mathrm{~S} \mathrm{~cm}^{-1}\right)$ is one order of magnitude higher than that of the pristine PEDOT:PSS film (4.64 $\mathrm{S} \mathrm{cm}^{-1}$ ). This change can be explained by the effect of $\pi-\pi$ interaction between PEDOT and MWCNTs. The electronic density transfer occurs from PEDOT to MWCNTs 
$\mathbf{a}$

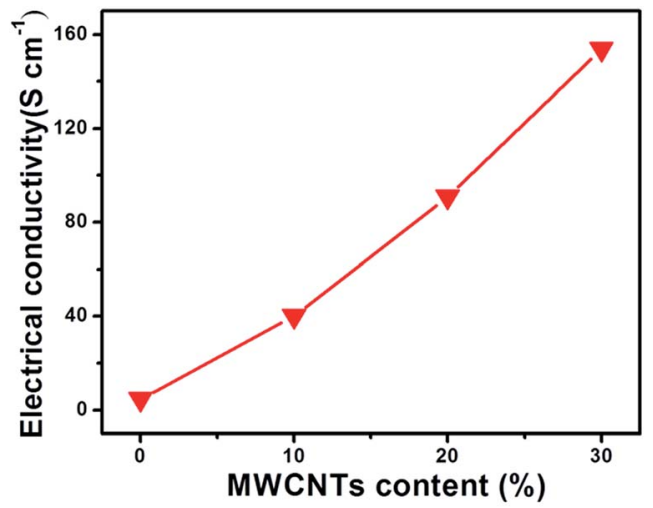

b

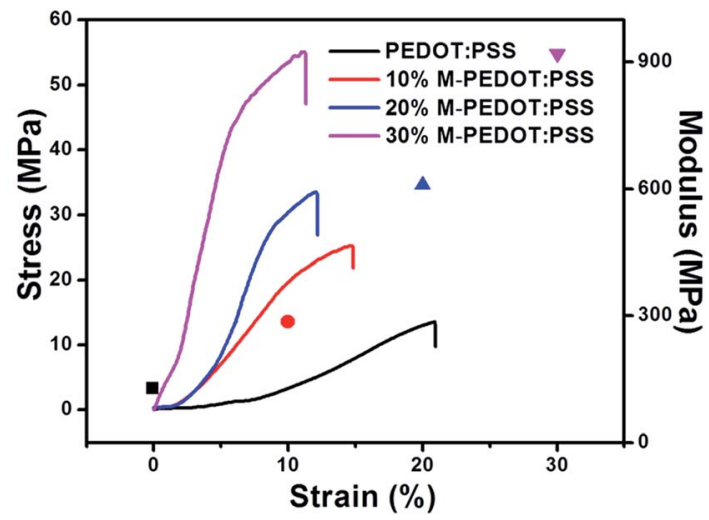

Fig. 3 (a) The electrical conductivity of composite films as a function of weight fraction of carboxylic MWCNTs. (b) Stress and modulus as a function of strain for the electrode membrane.

in M-PEDOT:PSS, therefore the charge becomes more delocalized on the PEDOT chains, which will be helpful to the charge transfer and conductivity enhancement of composite electrode films. ${ }^{22}$ The other reason for enhancing of electrical conductivity is a highly conductive MWCNTs network is formed in the polymer matrix with the MWCNTs weight fraction reaches a certain content. ${ }^{15}$

\subsection{Tensile test}

In order to expound the effect of carboxylic MWCNTs on mechanical properties of the electrode and actuator films, tensile test was performed and presented in Fig. $3 \mathrm{~b}$ and S2 (ESI). $\dagger$ Fig. $3 \mathrm{~b}$ shown the stress-strain curves of the pristine PEDOT:PSS and M-PEDOT:PSS electrode film with weight percentages of from $10 \%$ to $30 \%$. Pristine PEDOT:PSS electrode film fractured at maximum stress of $13.54 \mathrm{MPa}$, whereas MPEDOT:PSS film with weight percentages of $10 \%$ and $30 \%$ fractured at maximum stresses of $25.14 \mathrm{MPa}$ and $54.89 \mathrm{MPa}$ respectively. The calculated tensile modulus is listed in Table S2 (ESI). $\dagger$ The tensile modulus of the M-PEDOT:PSS electrode film (918.27 MPa) with a weight percentage of $30 \%$ is seven times that of the pristine PEDOT:PSS electrode film (127.64 MPa). It is noted that the elongation at break of the 30\% M-PEDOT:PSS electrode film attained only $11.27 \%$, which is almost half the PEDOT:PSS electrode film (20.93\%). According to the results we can see that the mechanical strength and stiffness of the flexible
PEDOT:PSS polymer are greatly enhanced by the addition of the rigid carboxylic MWCNTs. The greatly enhancements in tensile strength and modulus of the M-PEDOT:PSS electrode have a significant impact on the mechanical properties of obtained actuators. As shown in Fig. S2, $\uparrow$ the 30\% M-PEDOT:PSS electrode based actuator has a tensile strength of $7.75 \mathrm{MPa}$, which is $73.65 \%$ higher than that of the pristine PEDOT:PSS electrode based actuator. The calculated tensile modulus of the $30 \% \mathrm{M}$ PEDOT:PSS electrode based actuator is almost two times as much as the pristine PEDOT:PSS electrode based actuator (Table S2†).

\subsection{Electrochemical properties}

To evaluate the actuators' charge storage behavior, cyclic voltammetry (CV) was employed to display it. The thickness of the actuator is well controlled within the range between $108 \mu \mathrm{m}$ and $118 \mu \mathrm{m}$. Fig. 4a shown the electrochemical properties of the pristine PEDOT:PSS and 30\% M-PEDOT:PSS electrode based actuators examined by $\mathrm{CV}$ with a potential window from -2.5 to $2.5 \mathrm{~V}$ and a scan speed of $100 \mathrm{mV} \mathrm{s}^{-1}$. The shape of the $\mathrm{CV}$ curve for the pristine PEDOT:PSS electrode based actuators expressed as typical Faraday capacitor (FC) behavior, and the specific capacitance value which calculated from the integrated area of $\mathrm{CV}$ curves is $26.95 \mathrm{~F} \mathrm{~g}^{-1}$ as presented in Fig. $4 \mathrm{~b}$, which is close to the value reported by Terasawa. ${ }^{17}$ The $30 \%$ M-PEDOT:PSS electrode based actuators performed more electrical double layer
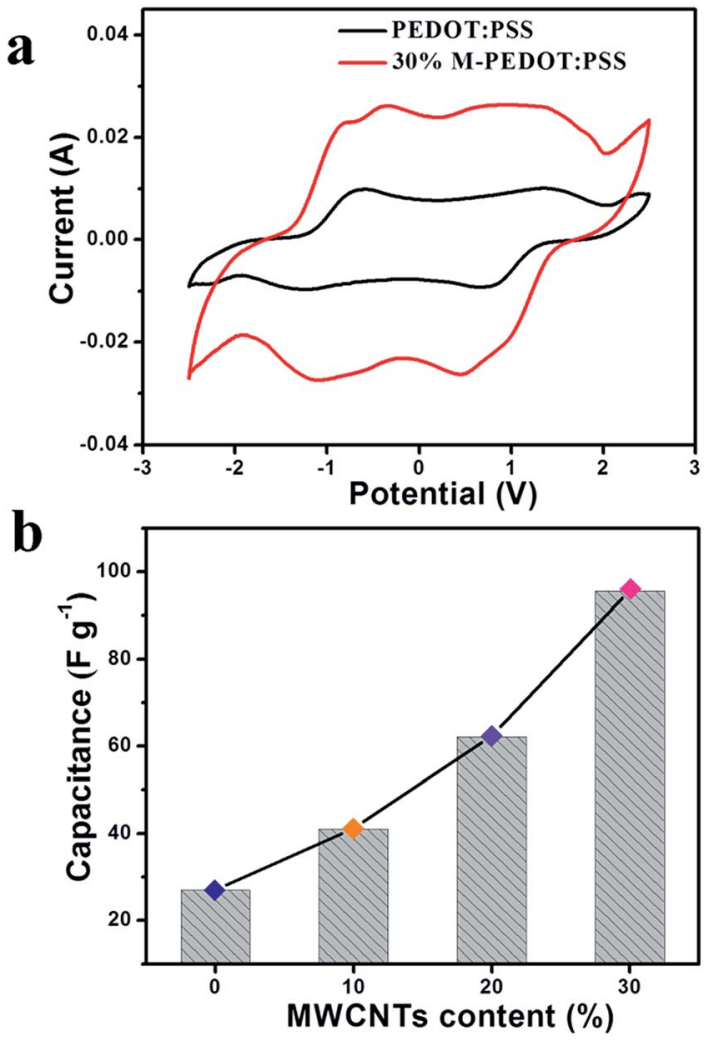

Fig. 4 (a) Cyclic voltammetry (CV) curves for the PEDOT:PSS and 30\% M-PEDOT:PSS electrode based actuators at $100 \mathrm{mV} \mathrm{s}^{-1}$ sweep rate. (b) The calculated capacitances for actuators. 
capacitor (EDLC) behavior except FC behavior owing to the addition of carboxylic MWCNTs. Because carbon nanotubes are regarded as ideal materials for electrochemical actuators based on the double-layer electrostatic mechanism. ${ }^{23}$ And the calculated specific capacitance value 30\% M-PEDOT:PSS electrode based actuators increases to $95.63 \mathrm{~F} \mathrm{~g}^{-1}$, with the EDLC mechanisms providing the greatest contribution. In general, the significantly improvement in capacitance is attributed to the addition of carboxylic MWCNTs. On the one hand, the 30\% M-PEDOT:PSS electrode owns high electrical conductivity which is helpful to acquire higher electrochemical energy storage capacity. On the other hand, the porous structure which formed after the addition of the carboxylic MWCNTs provides larger surface area and pore volume for adsorbing and accommodating ions, thus creating higher electrochemical performance. The electrochemical energy storage capacity plays a key role in emerging large bending deformation and blocking force of the actuator.

\subsection{Actuation performance}

The actuation performances of the actuators were examined with various conditions of harmonic electric inputs. The $30 \%$ M-PEDOT:PSS electrode based actuator performed symmetrical and large deformation (optical images in Fig. 1c) under the input voltage of $\pm 2.5 \mathrm{~V}$ at frequency of $0.1 \mathrm{~Hz}$. Fig. 5a introduced the responses of the two types actuators under the input voltage of $\pm 2.5 \mathrm{~V}$ at an frequency of $0.1 \mathrm{~Hz}$. The peak to peak displacement of the $30 \%$ M-PEDOT:PSS electrode based actuator is up to $15.7 \mathrm{~mm}$, which is about 2 times of PEDOT:PSS electrode based actuators. The high bending performance of the actuators is as a result of the high electrical, electrochemical and mechanical properties of the 30\% M-PEDOT:PSS electrode. Additionally, peak-to-peak displacements were measured under different step voltage from \pm 0.5 to $2.0 \mathrm{~V}$ peaks at $0.1 \mathrm{~Hz}$ frequencies. As shown in Fig. S3, $\uparrow$ The degree of bending deformation is roughly affected by the applied voltage. Since ionic mobility is highly dependent on the electric potential field in the polymer matrix. The peak-to-peak displacements of the $30 \%$ M-PEDOT:PSS electrode based actuator attained at $\pm 2 \mathrm{~V}$ is more than 3 times that at $\pm 0.5 \mathrm{~V}$. Moreover, the peak-to-peak strain of the actuators were also measured and calculated along with various excitation frequencies range from 0.1 to $10 \mathrm{~Hz}$. As shown in Fig. 5b, the bending performance of the $30 \%$ M-PEDOT:PSS electrode based actuator is impressively higher than pristine PEDOT:PSS electrode based actuator under all frequency conditions from 0.1 to $10 \mathrm{~Hz}$. The inset graph of Fig. $5 \mathrm{~b}$ revealed the harmonic responses of the $30 \% \mathrm{M}$ - $\mathbf{a}$
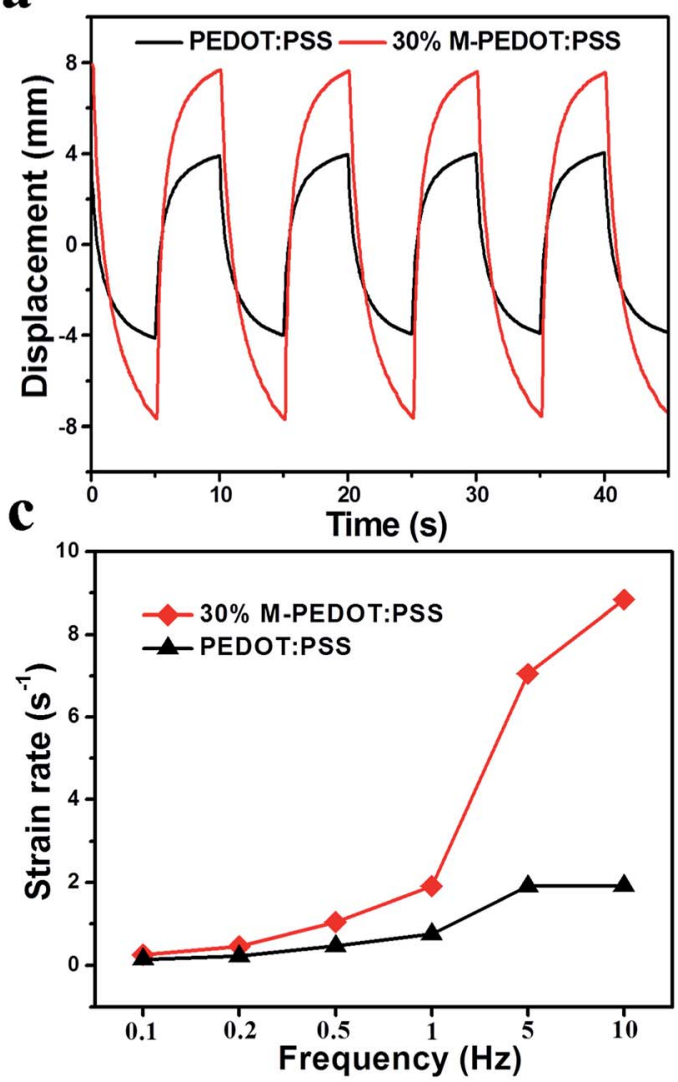

b

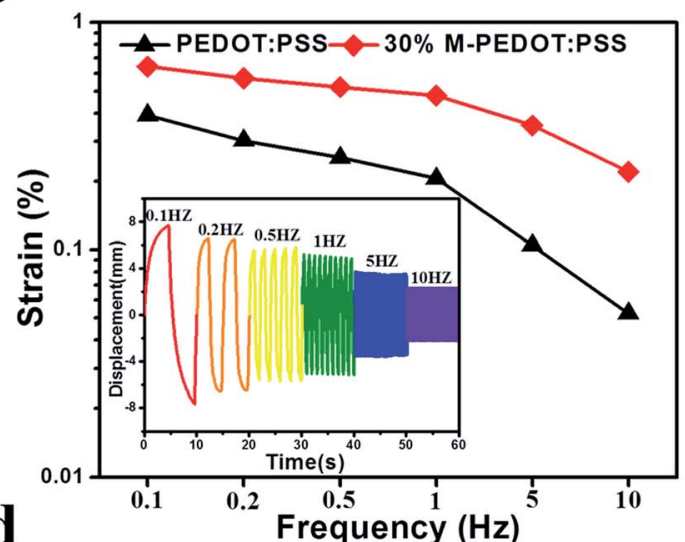

d

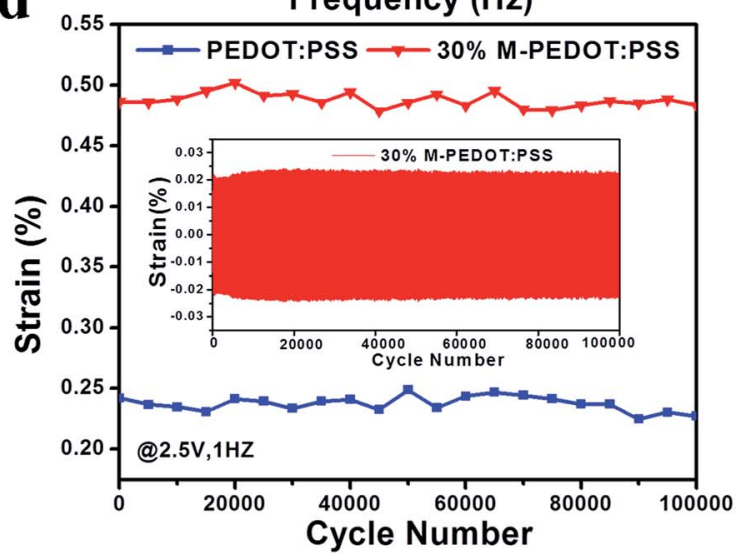

Fig. 5 Bending actuation performances of the PEDOT:PSS and 30\% M-PEDOT:PSS electrode based actuators (a) displacement under $2.5 \mathrm{~V}$ at $0.1 \mathrm{~Hz}$ (b) strain under $2.5 \mathrm{~V}$ from $0.1-10 \mathrm{~Hz}$ (c) strain rate under $2.5 \mathrm{~V}$ from $0.1-10 \mathrm{~Hz}$ (d) durability results of harmonic response for 100000 cycles under $2.5 \mathrm{~V}$ at $1 \mathrm{~Hz}$. 
PEDOT:PSS electrode based actuator with different excitation frequencies from 0.1 to $10 \mathrm{~Hz}$. As the frequency decreased, the tip displacement of the $30 \%$ M-PEDOT:PSS actuator gradually increased due to the sufficient time to move the inner electrolytes of the TPU polymer toward the electrodes. Fig. $5 \mathrm{c}$ was the calculated strain rate of two types actuators at different frequency from 0.1 to $10 \mathrm{~Hz}$. The strain rate of $30 \% \mathrm{M}$ PEDOT:PSS electrode based actuator is superior to that of the PEDOT:PSS electrode based actuators at high frequencies especially at $10 \mathrm{~Hz}\left(8.83 \% \mathrm{~s}^{-1}\right)$. In short, the porous structure of $30 \% \mathrm{M}$-PEDOT:PSS electrode led to easy and fast ion transport between the interfaces, thereby offering the capability to improve the bending actuation performance. Furthermore, the durability performance of the actuator was measured as seen in Fig. 5d. The 30\% M-PEDOT:PSS electrode based actuator demonstrated hardly any significantly performance degradation for 100000 cycles under an input voltage of $\pm 2.5 \mathrm{~V}$ at $1 \mathrm{~Hz}$. The strain of the $30 \% \mathrm{M}$-PEDOT:PSS electrode based actuator is maintained at as high as $99.4 \%$ compared to initial strain after 100000 cycles, while that of the PEDOT:PSS electrode based actuator is only about $93.8 \%$. This can be explained by the mechanical stability of electrode materials. In the process of actuation, the structure of PEDOT:PSS suffered certain damage due to frequent migration of ions. Therefore the strain of actuator decreased after 100000 cycles. But for M-PEDOT:PSS electrodes, the porous structure and synergy effect between PEDOT:PSS and carboxylic MWCNTs gave them high mechanical stability, which greatly improved cycle stability of actuator. As shown in the inset of Fig. 5d. The 30\% M-PEDOT:PSS electrode based actuator behaves excellent durability without significantly performance degradation over 100000 cycles. The excellent cycle stability of $30 \%$ M-PEDOT:PSS electrode based actuator is also attributed to the tightly integrated between the electrode layer and electrolyte layer by hot press method. The $30 \%$ M-PEDOT:PSS electrode of the actuator is completely bonded on the surface of the TPU membrane without delamination after 100000 cycles as shown in the cross-section SEM images in Fig. 1c.

The actuation performances enhancements of the $\mathrm{M}$ PEDOT:PSS electrode based actuators can be explained by actuation mechanism. As we all know, the dimensional changes of actuators based on CPs resulting from electrochemical doping, transportation of dopant ions between the polymer matrix and the surrounding electrolyte solution..$^{24}$ When a electric field is applied between two conducting polymer PEDOT:PSS electrode layers based actuator, the PEDOT $^{+}$PSS $^{-}$ ion pairs can be formed via the electrochemical doping induced by the potential difference between the positive and negative electrodes. ${ }^{25}$ And the cations or anions of the dissociated ionic liquid in TPU matrix will be absorbed into or excluded from the PEDOT:PSS electrode. In the positive electrode, the PEDOT network is oxidized and anions $\mathrm{BF}_{4}{ }^{-}$is incorporated inside the electrode according to the number of positive charges formed. Simultaneously, cations $\mathrm{EMI}^{+}$move and accumulate to the negative electrode driven by the electric field. Eventually, the actuator resulting in bending motion to the positive direction owing to the molecular size of cations $\mathrm{EMI}^{+}$is far larger than anions $\mathrm{BF}_{4}{ }^{-}$. For $30 \% \mathrm{M}$-PEDOT:PSS electrode based actuator, benefit from rough and porous structure of electrode, more ions in TPU matrix can be incorporated and accumulate into it when electric field is applied. It will lead to actuation performance enhancements of M-PEDOT:PSS electrode based actuator compared to PEDOT:PSS electrode based actuator.

\subsection{Efficiency of the conversion}

Energy conversion efficiency is an important parameter to evaluate performance of the actuator. In order to attain high conversion efficiency, the actuator need to convert a large proportion of the input electrical energy into mechanical work. According to previous reports, the electromechanical conversion efficiency of an actuator is mainly determined by the mechanical and electrical losses within the material. ${ }^{26}$ Liang et al. proposed a much detailed and convincing method to calculate the energy conversion efficiency of the actuator. ${ }^{27}$ The energy conversion efficiency from the electrical to mechanical $\left(\eta_{\text {transduction }}\right)$ of the actuator is calculated by the following equation:

$$
\eta_{\text {transduction }}=\frac{0.5 Y \varepsilon^{2} f \times \mathrm{Vol}}{V \times I_{\text {charge }}}
$$

where $Y$ is the Young's modulus, $\varepsilon$ is the bending strain, $f$ is the frequency of actuation, and $\mathrm{Vol}$ is the volume of the actuator, $V \times I_{\text {charge }}$ is the electric input power.

Fig. 6 was electromechanical conversion efficiency of the PEDOT:PSS and 30\% M-PEDOT:PSS electrode based actuators at different frequencies. According to equation, the calculated $\eta_{\text {transduction }}$ are mainly affected by the bending strain and tensile modulus of the actuators. Benefit from the higher bending strain, the conversion efficiency of the 30\% M-PEDOT:PSS electrode based actuators are greatly improved compared to PEDOT:PSS electrode based actuator. As the frequency increases from 0.1 to $10 \mathrm{~Hz}$, the conversion efficiency of the $30 \%$ M-PEDOT:PSS electrode based actuator significantly increased and reached to the maximum value $(1.04 \%)$ at $5 \mathrm{~Hz}$, which is almost 55 times compared to pristine PEDOT:PSS electrode

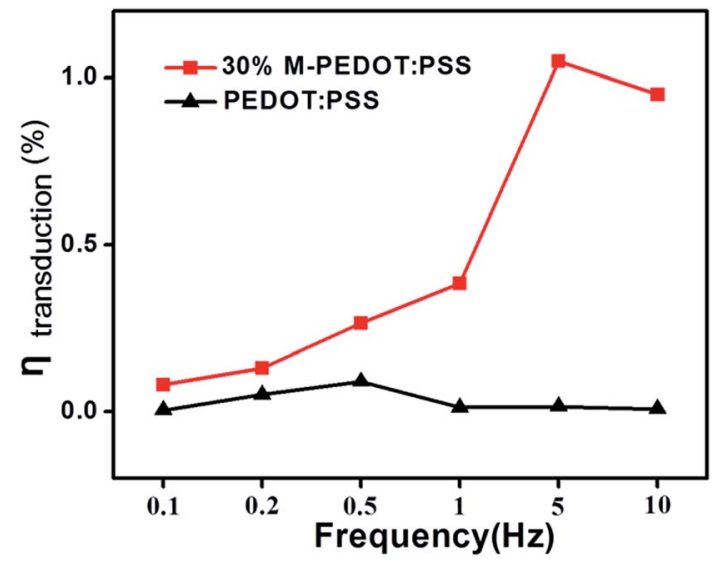

Fig. 6 Energy conversion efficiency of the PEDOT:PSS and 30\% MPEDOT:PSS electrode based actuators at different frequencies. 
based actuator at $5 \mathrm{~Hz}$. It is also noted that the conversion efficiency of pristine PEDOT:PSS electrode based actuator achieves the maximum value at $0.5 \mathrm{~Hz}$. This is because the $30 \% \mathrm{M}$ PEDOT:PSS electrode based actuator displays more slowly strain attenuation compared with pristine PEDOT:PSS electrode based actuator along with the increase of frequency (as shown in Fig. 5b). The greatly improvements of conversion efficiency are attributed to porous structure of M-PEDOT:PSS electrode, which accelerate the migration of ions and further promote the conversion from electric energy to mechanical energy. Our results shown the higher electromechanical conversion efficiency compared with the IPMC and other conducting polymer actuators. $^{27-31}$

\subsection{Blocking force}

The blocking force is an important parameter of the actuators because it is crucial for future engineering applications. It has been reported that actuator with higher stiffness (higher tensile strength and modulus) can generate higher blocking force. ${ }^{32}$ Fig. 7a shown the blocking force of the pure PEDOT:PSS and 30\% M-PEDOT:PSS electrode based actuators under the excitation of $\pm 2.5 \mathrm{~V}$ at frequency of $0.1 \mathrm{~Hz}$. The $30 \% \mathrm{M}$-PEDOT:PSS electrode based actuator has the highest blocking force 0.54 $\mathrm{mN}$, which is $193 \%$ enhancement than that of the pure PEDOT:PSS electrode based actuator $(0.15 \mathrm{mN})$. Fig. $7 \mathrm{~b}$ was the
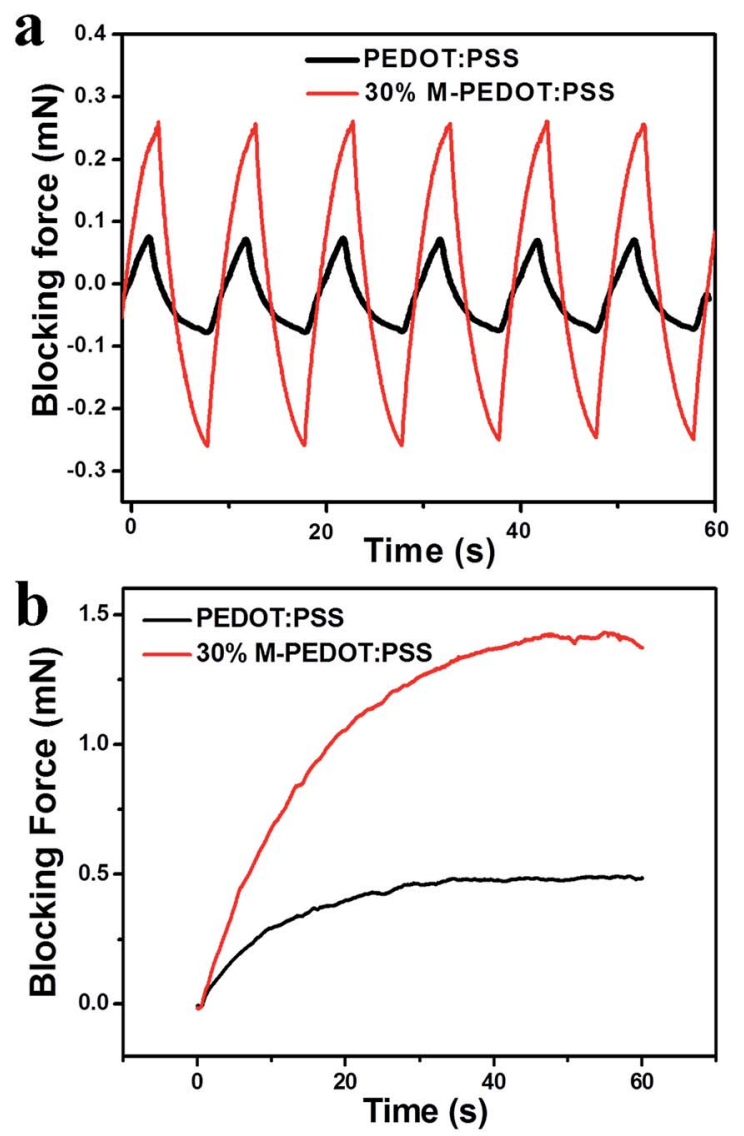

Fig. 7 Blocking force of PEDOT:PSS and 30\% M-PEDOT:PSS electrode based actuators (a) under $2.5 \mathrm{~V}$ at $0.1 \mathrm{~Hz}$. (b) under $2.5 \mathrm{~V} \mathrm{DC}$. blocking force of actuators under the excitation of 2.5 V DC. The blocking force of the $30 \%$ M-PEDOT:PSS electrode based actuator is up to $1.43 \mathrm{mN}$, which is three times that of the pure PEDOT:PSS electrode based actuator. The results demonstrates that the addition of carboxylic MWCNTs into PEDOT:PSS matrix significantly improve the blocking force of the actuator. We attributed the highly enhanced output force of the $30 \% \mathrm{M}$ PEDOT:PSS electrode based actuator to the high tensile modulus and electrochemical properties caused by $\mathbf{M}$ PEDOT:PSS electrodes.

\section{Conclusion}

In this paper, we developed a high-performance electrochemical actuator based on M-PEDOT:PSS electrode. The good compatibility of PEDOT:PSS with MWCNT through $\pi-\pi$ interaction and hydrogen bond provided much higher stiffness and strength for the M-PEDOT:PSS electrodes. In addition, the porous structure and high electric conductivity of $\mathrm{M}$ PEDOT:PSS electrode could facilitated ion transmission and accumulation into it, which played a key role in improving actuation performance. The obtained M-PEDOT:PSS electrode based actuators possessed superior electro-chemo-mechanical properties and exceptionally high actuation performance compared with pure PEDOT:PSS actuators. It is found that the M-PEDOT:PSS electrode based actuator performed higher tensile modulus (82.18 MPa) and specific capacitances (95.63 F $\mathrm{g}^{-1}$ ) with increasing the MWCNT content from $0 \mathrm{wt} \%$ to 30 wt $\%$. Furthermore, the $30 \%$ M-PEDOT:PSS electrode based actuator are capable of producing large bending deformation (up to the strain of $0.64 \%$ ), high strain rates $\left(8.83 \% \mathrm{~s}^{-1}\right)$ and greatly force generation $(1.43 \mathrm{mN})$ at $2.5 \mathrm{~V}$. Beyond that, superb energy conversion efficiency $(1.04 \%)$ of electrical input to mechanical output is obtained for the M-PEDOT:PSS electrode based actuators, which shows great promise in the development of next generation electroactive polymer actuator and practical application.

\section{Acknowledgements}

This work was supported by the National Natural Science Foundation of China (Grant No. 21373263), the External Cooperation Program of BIC from Chinese Academy of Sciences (Grant No. 121E32KYSB20130009), the Science and Technology of Jiangsu Province (Grant No. BE 2016086), and the Special Project of Nanotechnology in Suzhou (ZXG201423).

\section{References}

1 E. Smela, Adv. Mater., 2003, 15, 481-494.

2 Y. Bar-Cohen, Proc. Inst. Mech. Eng., Part C, 2007, 221, 11491156.

3 G. Wu, Y. Hu, J. Zhao, T. Lan, D. Wang, Y. Liu and W. Chen, Small, 2016, 12, 4986-4992.

4 I. S. Romero, N. P. Bradshaw, J. D. Larson, S. Y. Severt, S. J. Roberts, M. L. Schiller, J. M. Leger and A. R. Murphy, Adv. Funct. Mater., 2014, 24, 3866-3873. 
5 F. Carpi and E. Smela, Biomedical applications of electroactive polymer actuators, John Wiley \& Sons, 2009.

6 A. Maziz, C. Plesse, C. Soyer, C. Chevrot, D. Teyssie, E. Cattan and F. Vidal, Adv. Funct. Mater., 2014, 24, 4851-4859.

7 J. Huang, P. Miller, J. De Mello, A. De Mello and D. Bradley, Synth. Met., 2003, 139, 569-572.

8 Q. S. Wei, M. Mukaida, Y. Naitoh and T. Ishida, Adv. Mater., 2013, 25, 2831-2836.

9 M. Dobbelin, R. Marcilla, M. Salsamendi, C. Pozo-Gonzalo, P. M. Carrasco, J. A. Pomposo and D. Mecerreyes, Chem. Mater., 2007, 19, 2147-2149.

10 J. Y. Oh, M. Shin, J. B. Lee, J. H. Ahn, H. K. Baik and U. Jeong, ACS Appl. Mater. Interfaces, 2014, 6, 6954-6961.

11 Y. J. Xia, K. Sun and J. Y. Ouyang, Adv. Mater., 2012, 24, 24362440.

12 N. Kim, S. Kee, S. H. Lee, B. H. Lee, Y. H. Kahng, Y. R. Jo, B. J. Kim and K. Lee, Adv. Mater., 2014, 26, 2268-2272.

13 S. Iijima, Nature, 1991, 354, 56-58.

14 J. Park, A. Lee, Y. Yim and E. Han, Synth. Met., 2011, 161, 523-527.

15 J. A. Li, J. C. Liu and C. J. Gao, J. Polym. Res., 2010, 17, 713718.

16 K.-S. Kim and S.-J. Park, Mater. Sci. Eng., B, 2011, 176, 204209.

17 N. Terasawa and K. Asaka, Langmuir, 2016, 32, 7210-7218.

18 M. Kotal, J. Kim, K. J. Kim and I. K. Oh, Adv. Mater., 2016, 28, 1610-1615.

19 L. Kong and W. Chen, Adv. Mater., 2014, 26, 1025-1043.
20 J. Li, W. Ma, L. Song, Z. Niu, L. Cai, Q. Zeng, X. Zhang, H. Dong, D. Zhao, W. Zhou and S. Xie, Nano Lett., 2011, 11, 4636-4641.

21 Y. Li, R. Tanigawa and H. Okuzaki, Smart Mater. Struct., 2014, 23, 074010.

22 H. Shi, C. C. Liu, Q. L. Jiang and J. K. Xu, Adv. Electron. Mater., 2015, 1, 1500017.

23 W. Lu, A. G. Fadeev, B. H. Qi, E. Smela, B. R. Mattes, J. Ding, G. M. Spinks, J. Mazurkiewicz, D. Z. Zhou, G. G. Wallace, D. R. MacFarlane, S. A. Forsyth and M. Forsyth, Science, 2002, 297, 983-987.

24 S. S. Kim, J. H. Jeon, C. D. Kee and I. K. Oh, Smart Mater. Struct., 2013, 22, 085026.

25 S. S. Kim, J. H. Jeon, H. I. Kim, C. D. Kee and I. K. Oh, Adv. Funct. Mater., 2015, 25, 3560-3570.

26 R. E. Pelrine, R. D. Kornbluh and J. P. Joseph, Sens. Actuators, A, 1998, 64, 77-85.

27 P.-J. Cottinet, C. Souders, D. Labrador, S. Porter, Z. Liang, B. Wang and C. Zhang, Sens. Actuators, A, 2011, 170, 164-171.

28 Y. Wang, H. Chen, Y. Wang, B. Luo, L. Chang, Z. Zhu and B. Li, Polym. Eng. Sci., 2014, 54, 818-830.

29 G.-H. Feng and J.-W. Tsai, Smart Mater. Struct., 2011, 20, 015027.

30 K. Kaneto, H. Fujisue, M. Kunifusa and W. Takashima, Smart Mater. Struct., 2007, 16, S250-S255.

31 R. Temmer, I. Must, F. Kaasik, A. Aabloo and T. Tamm, Sens. Actuators, B, 2012, 166-167, 411-418.

32 H.-T. Lim, J.-W. Lee and Y.-T. Yoo, J. Korean Phys. Soc., 2006, 49, 1101-1106. 